\title{
New Analyses of Double-Bang Events in the Atmosphere
}

\author{
Célio A. Moura* and Marcelo M. Guzzo ${ }^{\dagger}$ \\ Instituto de Física Gleb Wataghin - UNICAMP \\ Cx.P.6165 13083-970 Campinas, SP Brazil
}

Received on 23 November, 2007

\begin{abstract}
We use CORSIKA+Herwig simulation code to produce ultra-high energy neutrino interactions in the atmosphere. Our aim is to reproduce extensive air showers originated by extragalactic tau-neutrinos. For charged current tau-neutrino interactions in the atmosphere, beside the air shower originated from the neutrino interaction, it is expected that a tau is created and may decay before reaching the ground. That phenomenon makes possible the generation of two related extensive air showers, the so called Double-Bang event. We make an analysis of the main characteristics of Double-Bang events in the atmosphere for mean values of the parameters involved in such phenomenon, like the inelasticity and tau decay length. We discuss what may happen for the "out of the average" cases and conclude that it may be possible to observe this kind of event in ultra-high energy cosmic ray observatories such as Pierre Auger or Telescope Array.
\end{abstract}

Keywords: Neutrinos; Astroparticles; Ultra-high energy; Atmosphere; Fluorescence detector

\section{INTRODUCTION}

It is believed that neutrinos with energies of the order of $10^{18} \mathrm{eV}(1 \mathrm{EeV})$ arrive at the Earth from extragalactic sources and may interact with the nuclei in the atmosphere generating cascades of particles called Extensive Air Showers (EASs) $[1,2]$. There are several techniques to measure EASs, but for energies above $1 \mathrm{EeV}$ basically two of them are being used: one is to detect the particles when they reach the ground through water tanks equipped with photomultipliers [3-5]. The photomultipliers are used to detect the Cherenkov light emitted by the ultra relativistic particles when they cross the water inside the tanks. By spreading the Cherenkov tanks in a big and plane area it is possible to determine the distribution of particles when the EAS reach the ground and with this information to determine the direction and energy of the incident particle that generated the event. The other technique is to observe the scintillation light of the atoms which are excited during the developing process of the EAS [4-6]. The atoms emit detectable visible and ultraviolet light when they return to their ground states. The detectors used in this method are called Fluorescence Detectors (FDs) because of the similarity of the scintillation with the fluorescence light. This technique of detection can be used to measure the profile of the EAS when it develops through the atmosphere.

To distinguish which particle have generated an EAS is not an easy task, but we can take advantage of the different depth of the atmosphere depending on the zenith angle. While for vertical angles the atmospheric depth is approximately $1000 \mathrm{~g} / \mathrm{cm}^{2}$, for horizontal angles it is approximately $36000 \mathrm{~g} / \mathrm{cm}^{2}$. Ordinary cosmic rays like protons, electrons, photons, helium nucleus or heavier nuclei, interact in the top of the atmosphere and when they come from almost horizon-

*moura@ifi.unicamp.br ; Also at Departamento de Física, Centro de Investigación y de Estudios Avanzados del IPN. Apdo. Postal 14-740 07000 México DF, Mexico.

$\dagger$ Electronic address: guzzo@ifi.unicamp.br tal angles the EAS generated arrives at the detector basically only with the muonic component, because the hadronic component is extinguished and the electromagnetic one is almost completely absorbed by the atmosphere [7, 8]. Neutrinos, on the other hand, because of their very low cross section, are the only particles beside the muons that can go deep in the atmosphere before interacting. In this way it is possible that neutrinos coming from very near horizontal angles interact close to the detector.

The case of tau-neutrinos is even more special. Because it is not expected that tau-neutrinos are created in extragalactic sources, i.e., tau-neutrinos are due to neutrino oscillation during the propagation from the source to the detector $[9,10]$, and, what is the subject of this paper, because when the tauneutrino interacts via charged current $(\mathrm{CC})$ in the atmosphere it creates an EAS which contains a tau. For energies of the order of $1 \mathrm{EeV}$, the tau decays in a distance comparable to the size of the EAS and almost all of the times it generates another EAS that may also be detectable. This kind of unique signature, with two EASs coming from the same direction in a time interval of the order of the tau mean lifetime, is named Double-Bang event.

The detection of a Double-Bang would be very important both from the astrophysical and the particle physics point of view. Because this event, as already mentioned, has a unique Ultra-High Energy (UHE) tau-neutrino signature given by two EASs coming from the same direction in a time interval corresponding to the tau decay length, with an approximately determined energy relation, it can be used to test the existence of the UHE tau-neutrinos. On the other hand the non observation of Double-Bang events together with the Earth Skimming $[8,11-14]$ events can help to set an upper limit for the UHE tau-neutrino flux. The future data of the Pierre Auger Observatory [15] for inclined EASs, that means EASs coming from zenith angles bigger than $60^{\circ}$, will be of fundamental importance on this analysis. The suppression of the cosmic ray flux above the GZK cutoff does not apply for neutrinos however the source anisotropy recently measured [16] can indicate some nearby UHE neutrino sources, and theoretical models 
that relate the fluxes of UHE neutrinos and cosmic rays can be tested, as well as UHE neutrino oscillations.

We present simulations of Double-Bang events generated in the atmosphere by tau-neutrinos with energies of $0.5 \mathrm{EeV}$, $1.5 \mathrm{EeV}$ and $5 \mathrm{EeV}$. The inelasticity for the simulated events is approximately $0.2,0.73$, and 0.92 for each energy respectively, the tau decay length is of $20 \mathrm{~km}$ and the energy fraction from the tau that goes into the second EAS is of $2 / 3$. We also discuss cases with different energies, decay lengths, inelasticities, and second EAS's energy. Because for ultra-high energies the neutrino cross section, inelasticity, and flux are still uncertain, and because in our analysis we do not use any specific detector characteristic, we do not calculate the DoubleBang event rate for neutrinos interacting in the atmosphere. The characteristics we observe from the simulations confirm that Double-Bang events can develop in the atmosphere and FDs of experiments like Pierre Auger [17], HiRes and Telescope Array [18] may detect them depending on the neutrino flux and cross section, as it was pointed out in [19].

\section{ULTRA-HIGH ENERGY NEUTRINO INDUCED EVENTS}

Consider a neutrino arriving at the Earth and interacting via $\mathrm{CC}$ with a nucleon in the atmosphere generating a charged lepton and other fragments:

$$
\mathrm{v}_{l}+N \rightarrow l+X
$$

where $l$ is the lepton flavor $(e, \mu, \tau)$. For a neutral current (NC) interaction another neutrino is created rather then a charged lepton.

After the CC interaction, the neutrino energy is divided between the charged lepton and the other fragments that generate the EAS in the following way:

$$
E_{\mathrm{v}}=E_{1}+E_{l}
$$

where $E_{\mathrm{V}}$ is the incident neutrino energy, $E_{1}$ is the energy deposited in the fragments that generate the EAS and $E_{l}$ is the charged lepton energy.

Because the energy distribution varies for each interaction, it is interesting to define the inelasticity, that is the fraction of the neutrino energy that goes to the EAS and not to the charged lepton. The inelasticity is:

$$
y=\left(E_{\mathrm{v}}-E_{l}\right) / E_{\mathrm{v}} .
$$

Combining Eqs. (2) and (3) we have:

$$
E_{1}=y E_{\mathrm{v}}
$$

and finally from Eqs. (2) and (4) we find the neutrino energy fraction transfered to the charged lepton:

$$
E_{l}=(1-y) E_{v} .
$$

When a muon-neutrino interacts via $\mathrm{CC}$, it creates an EAS with the same characteristics of the ones produced via NC interactions for any neutrino flavor. It is because similarly to the neutrinos created after the NC interactions, the muon created after a CC muon-neutrino interaction almost does not interact with the atmosphere [20] and for the energies considered, the muon decay length is much longer than its interaction length.

Electron and tau-neutrino $\mathrm{CC}$ interactions are completely different. The electron, created after the electron-neutrino interaction, interacts immediately generating a cascade of electromagnetic particles beside the hadronic component generated by the other fragments created in the first interaction. The tau created after the tau-neutrino interaction propagates in a very similar way to the muon, but its mean lifetime is much shorter. For the energies considered the decay length of the tau is of the order of few tens of $\mathrm{km}$ in the laboratory frame, comparable to the length of the hadronic EAS created. When the tau decays, it may generate a second hadronic shower and both showers together generate a sign characteristic of tauneutrino interactions only.

\section{TAU-NEUTRINOS INTERACTING IN THE ATMOSPHERE}

Many articles have been published analyzing the potential of the Pierre Auger Observatory to detect and investigate neutrinos [21, 22] and specially Earth Skimming events. In reference [14] down-going tau-neutrino events are also investigated. According to the estimated neutrino flux coming from Active Galactic Nuclei [23, 24], Gamma Ray Bursts [25], Topological Defects [24, 26], and Cosmogenic Neutrinos $[27,28]$ as well as to the neutrino cross section extrapolated to ultra-high energies [29], it is expected a number of tauneutrino events in Auger approximately of the order of between 0.1 and 1 event per year, with energies around $1 \mathrm{EeV}$.

Based on the propose of John Learned and Sandip Pakvasa to detect tau-neutrinos with energies of the order of $1 \mathrm{PeV}$ in detectors under water or ice [9], it has been proposed to use FDs to detect tau-neutrinos with energies of the order of $1 \mathrm{EeV}$ interacting in the atmosphere $[19,30]$. Here we investigate through simulations the properties of the Double-Bang events in the atmosphere. These properties do not depend on the detector, despite we are concerned with the characteristics as efficiency and field of view for FDs.

After it is created the tau propagates on average, before decaying in the laboratory frame, a distance given by:

$$
\langle L\rangle=\gamma c \tau,
$$

where $\gamma$ is the Lorentz factor and $\tau$ is the tau mean lifetime. In terms of the energy given in units of $\mathrm{EeV}$, we have that the distance traveled by the tau in the laboratory frame is, on average: 


$$
\begin{aligned}
\langle L\rangle & \simeq \frac{E_{\tau}}{[\mathrm{EeV}]} \times 49 \mathrm{~km}, \\
& \simeq(1-y) \frac{E_{\mathrm{v}}}{[\mathrm{EeV}]} \times 49 \mathrm{~km} .
\end{aligned}
$$

In our simulations we consider only the case of hadronic decay, despite we could have considered also the electronic decay $\left(\tau \rightarrow e v_{e} \nu_{\tau}\right)$, which corresponds to almost $18 \%$ of the tau decay branching ratio [31] and produce a electromagnetic shower with, on average, about $1 / 3$ of the tau energy. Only the hadronic branching ratio is responsible for $64 \%$ of the tau decays. Based on the hadronic branching ratio of the tau decay, that is basically decay to pions, and considering that each decay product carries on average the same amount of energy, we calculate that the energy of the second EAS, originated by the tau decay, is on average:

$$
\begin{aligned}
\left\langle E_{2}\right\rangle & \approx \frac{2}{3} E_{\tau}, \\
& \approx \frac{2}{3}(1-y) E_{v} .
\end{aligned}
$$

This expression is confirmed by simulations [32].

\section{SIMULATIONS}

Due to the low event rate expected for the neutrinos, combined with the limitations of the detection and discrimination techniques, there is no measured UHE EAS that could be uniquely associated to a primary neutrino. Monte Carlo simulations are an important tool to study the characteristics of UHE events that may be generated by neutrinos. In general, the EAS longitudinal development depends on the energy and the type of the primary particle, as well as on the interaction depth and the incident angle in the atmosphere. Until this moment, the only way to study in a systematical way the longitudinal development of the charged particles in a Double-Bang event is through simulations.

A study of the characteristics of the UHE EASs generated by electron and muon-neutrinos was made in [33] through CORSIKA [34] simulations. CORSIKA versions 6.203 and 6.204 themselves, that we used to do the simulations here, are not able to simulate neutrinos as primary particles, so the neutrino interaction is made by Herwig [35] and then, the results of this interaction are taken by CORSIKA as the primary particles which give rise to the EAS. In CORSIKA, the hadronic interaction models used were QGSJET [36] for high energies and GHEISHA [37] for low energies $\left(<10^{11} \mathrm{eV}\right)$. We used a thinning of $\varepsilon_{t h}=10^{-6}$, what permits the program to follow all the particles with energies down to $10 \mathrm{GeV}$. The energy cutoffs were $0.05,0.05,0.0001$, and $0.0001 \mathrm{GeV}$ for hadrons, muons, electrons and photons respectively. Until the present moment, simulations with tau-neutrino as primary particle and the decay of taus in CORSIKA are not available. Because of that we simulate Double-Bang events through phenomenological arguments, using muon neutrinos as primary particles and pions as the generators of the EAS which in principle may come from the tau decay. A more accurate and particular analysis for Auger, e.g., can be done using the method described in [14], specially for the simulation of tau decays with the software TAUOLA [32] and the light propagation and the hardware detector trigger simulated by means of the Auger software framework called Offline [38]. We want to be more general and to do simulations specific for Auger is beyond the scope of this paper.

Fig. 1 presents the different behavior of an EAS depending on the type of interaction and the inelasticity value. In each of the graphics of the figure we have 50 EASs induced by muon-neutrino with energy $E=0.5 \mathrm{EeV}$ and incident angle of $75^{\circ}$. The dashed lines are the Gaisser-Hillas fit [39] to the average of all the EASs in a graphic. The discussion here can be thought to be naive, but it is very useful to show that the simulation code is producing, on average, what is expected in the framework of the Standard Model.

On the left side of the Fig. 1 one has CC and NC interactions happening freely. Also the elasticity value is obtained randomly by Monte Carlo method with CORSIKA. We obtained $35 \mathrm{CC}$ interactions (black continuous lines) and $15 \mathrm{NC}$ interactions (blue dotted lines). As it is expected because of the bigger cross section, $70 \%$ of the interactions are CC interactions.

On the central part we present only CC interactions. There are two showers with bigger number of charged particles than in the simulations presented on the left side, but it may probably be because of statistical fluctuations. On average, for tau and muon-neutrino induced EASs, one expects the same longitudinal profile for both $\mathrm{CC}$ and NC interactions. The difference as we have seen, beside the cross section difference between $\mathrm{CC}$ and $\mathrm{NC}$, is that in the case of $\mathrm{CC}$ a charged lepton is created and for $\mathrm{NC}$ it is a neutrino.

The right side of the Fig. 1 has only EAS's generated via $\mathrm{CC}$ interaction and with inelasticity $y=0.2 \pm 0.05$. Because the most part of the energy goes to the charged lepton, the EAS with hadronic nature has low energy. Moreover, because the inelasticity is basically fixed around one value, the maximum number of charged particles is concentrated around a fixed value too. For that value of inelasticity, the number of charged particles is around $10^{8}$ particles. The Gaisser-Hillas function gives a good approximation with low deviation in this case. We can see that, as expected for these energies [40], for the two previous cases the average value of $y$ is nearly the same, but with much bigger deviation from the average since the inelasticity is a free parameter chosen randomly by the simulation code.

\section{A. Double-Bangs}

The alternative we use to simulate events generated by tauneutrinos in the atmosphere is to input muon-neutrino as primary particle. The main characteristics of muon and tauneutrino induced events are the same with the exception that a muon-neutrino interacting via $\mathrm{CC}$ generates a muon. As we are simulating tau-neutrino interactions, we need the creation 

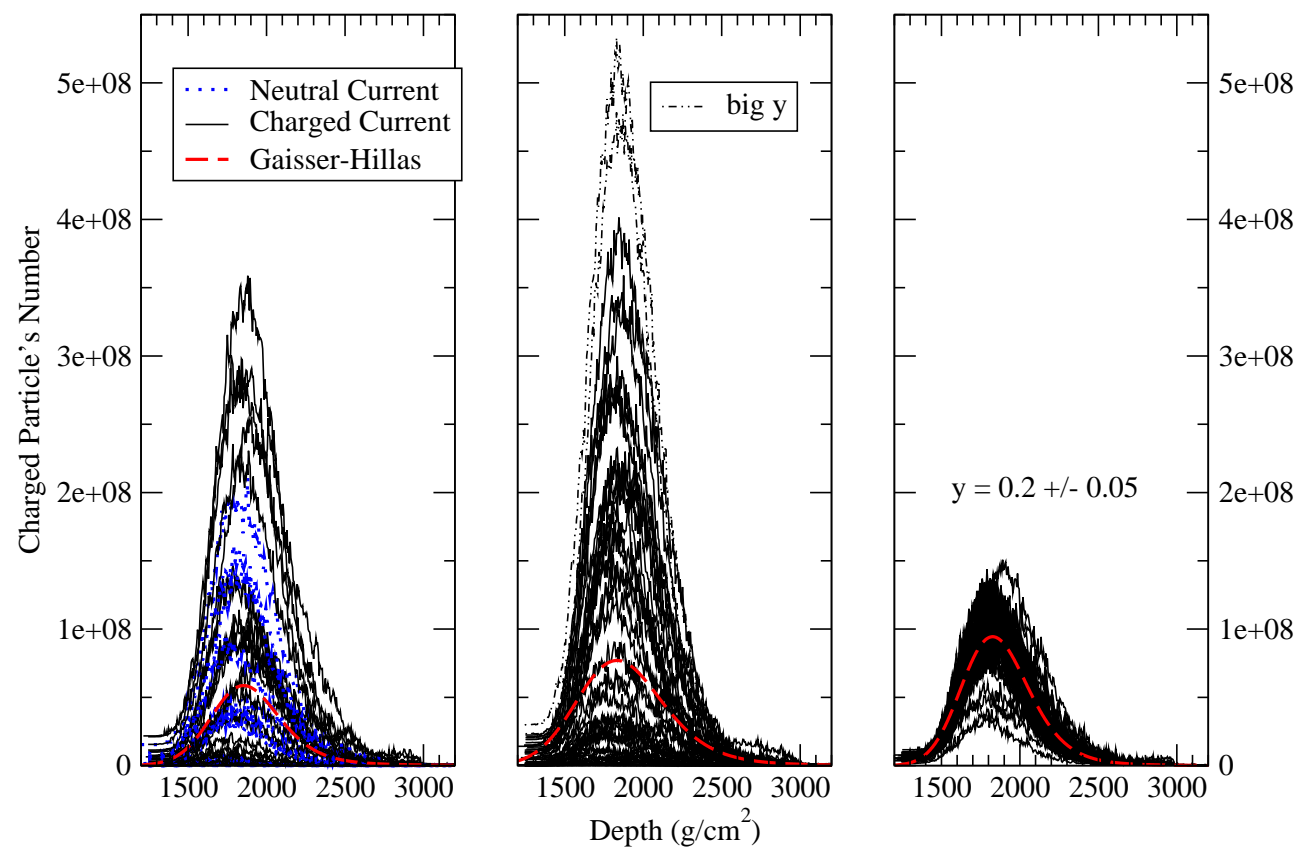

FIG. 1: Charged particles as a function of atmospheric depth. Comparison of the kind of interaction of muon-neutrino induced events with energy $E=0.5 \mathrm{EeV}$. In the left side we have interactions randomly induced via Monte Carlo. In the center only CC interactions are present, and in the right side we have CC interactions with inelasticity $y=0.2 \pm 0.05$. Read text for details.

of a tau. To simulate the tau creation and subsequent decay we use pions. One can divide the tau decay in two main modes: $64 \%$ for the hadronic modes and $36 \%$ for the leptonic modes. Despite the leptonic decay mode may generate a electromagnetic shower $18 \%$ of the times as we discuss in Section III, in our simulations we consider only the hadronic modes in which the main decay products are pions. In this way we generate the second shower inputing the pion as primary particle in the same direction of the muon-neutrino, after a distance $L$, from Eqs. (7)-(8), corresponding to the tau mean lifetime and with the corresponding energy $E_{2}$ from Eqs. (9)-(10).

In Figs. 2, 3, and 4 we show events for the tau energy of approximately $0.4 \mathrm{EeV}$. It means that on average the tau should have run a distance of about $20 \mathrm{~km}$ from the neutrino interaction point until the tau decay, and considering, from Eq. (9), that $2 / 3$ of the tau energy goes to the decay products, it corresponds to a pion energy of $0.27 \mathrm{EeV}$. Each graphic of the figures contain 10 Double-Bang events, that is to say 10 showers initiated by muon-neutrino and 10 initiated by pion $\left(\pi^{-}\right)$. The distance, in the neutrino propagation axis, from the neutrino interaction point to the ground is represented by $l$.

In the bottom left of Fig. 3 we have a kind of fake DoubleBang event where the second EAS was not generated by the tau decay, but by some fragment of the first EAS that interacted or decayed after traveling a very long distance comparing with the mean distance traveled by the taus with $0.4 \mathrm{EeV}$ energy. We checked that this second generated EAS is of electromagnetic characteristic and it was probably generated by a muon decay. It is important to notice that among the 310 simulations initiated by neutrino that we made, in only one it happened to be produced a second shower imitating the sec- ond bang of a Double-Bang event. It would be a background of less than $0.5 \%$ of the total number of events. Adding to that, the fake Double-Bang is out of the average and with an electromagnetic second Bang, what completely distinguish them from the case we are studying with hadronic second Bangs.

To obtain the events generated by $0.5 \mathrm{EeV}$ neutrinos depicted in Figs. 2 and 3 we use only inelasticity values of $y=0.2 \pm 0.05$. In this way we have, from Eq. (4), $E_{1} \sim E_{\mathrm{v}} / 5$, and also from Eq. (10), $E_{2} \approx 2.67 E_{1}$. Analyzing events coming from different angles and interaction depths, we may say which values of theses parameters are more favorable for detecting Double-Bang events with energies of about $0.5 \mathrm{EeV}$ and $20 \%$ of inelasticity.

In Fig. 4 we have events with more energy for the primary neutrino. In this case, due to the energy distribution between the first EAS and the charged lepton, we have the first EAS more energetic than the second one. For $E_{\mathrm{v}}=1.5 \mathrm{EeV}$ and $y=0.73, E_{1}=1.1 \mathrm{EeV} \approx 4 E_{2}$. If the neutrino energy is $E_{\mathrm{v}}=$ $5 \mathrm{EeV}$ and $y \approx 0.92$, than $E_{1}=4.6 \mathrm{EeV} \approx 17 E_{2}$.

Observing the simulations in Figs. 2 and 3 we have the hint that the bigger is the incident angle, for neutrinos interacting always at the same distance from the detector, the bigger is the distance between the two maximums of the generated EASs. Furthermore, the same happens if the distance from the primary interaction to the detector diminishes. We conclude that the worse situations to detect Double-Bangs generated by neutrinos with energies of the order of $0.5 \mathrm{EeV}$ and inelasticity of about $20 \%$ are for $l=50 \mathrm{~km}$ and $\theta=50^{\circ}$, as well as for $l=30 \mathrm{~km}$ and $\theta=80^{\circ}$. In the first situation the two EASs overlap each other and in the second one, the second EAS reach the ground before it has a considerable number 


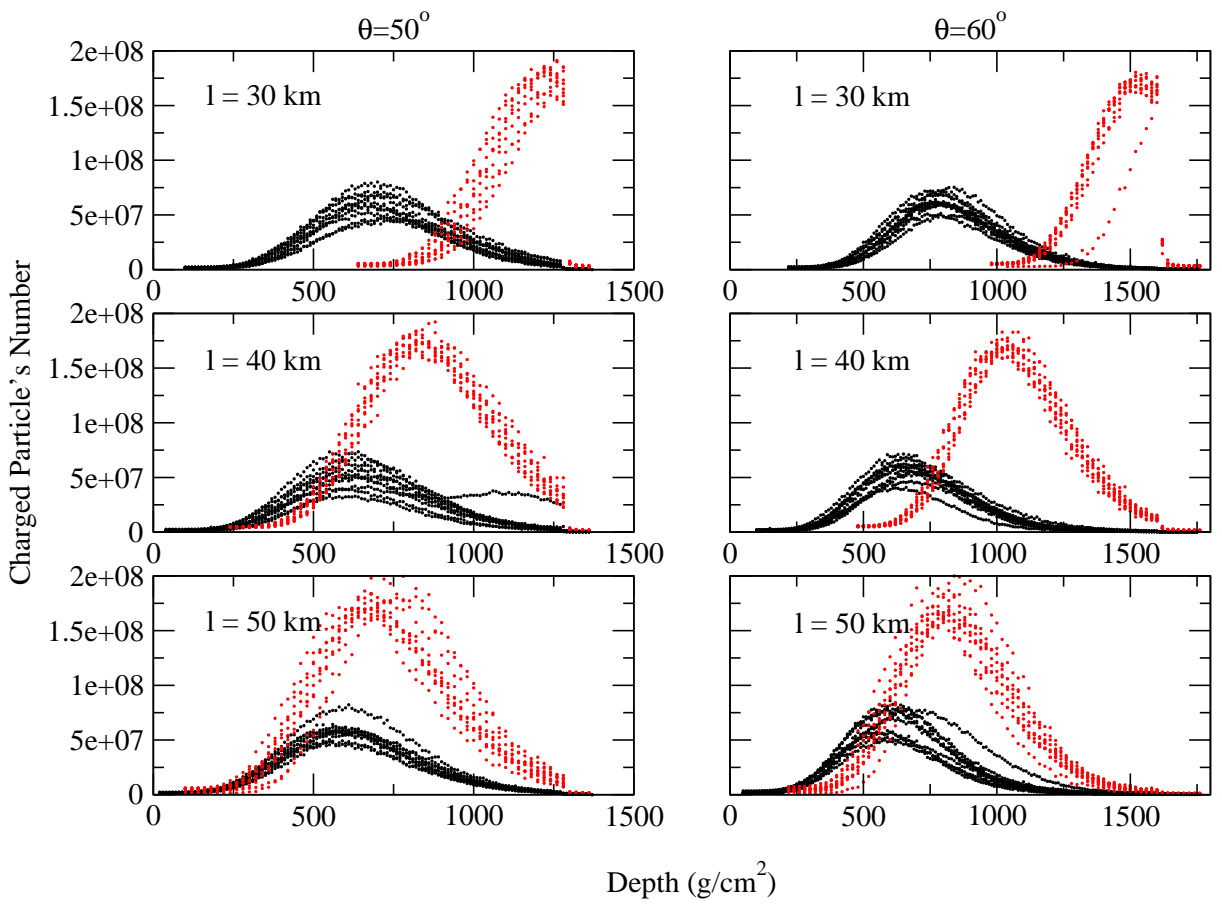

FIG. 2: Double-Bangs generated by tau-neutrinos in the atmosphere. Each graphic contains ten events represented by the number of charged particles as a function of depth with primary neutrino energy of $0.5 \mathrm{EeV}$. The incident angle is of $50^{\circ}$ in the first column and $60^{\circ}$ in the second one. The interaction distance to the ground is $l=30,40$ and $50 \mathrm{~km}$ respectively for each line. The first EASs, with less than $10^{8}$ charged particles, are represented in black and the second ones, due to the tau decay with more than the double number of particles, are represented in red.

of particles. These effects may happen due to the different atmospheric density depending on the height. Close to the surface of the Earth, where the density is higher, the particles have more probability of interacting and consequently the longitudinal development of the EAS is faster.

\section{DISCUSSION AND SUMMARY}

UHE neutrinos probably coming from extragalactic sources may interact in the atmosphere via $\mathrm{NC}$ or $\mathrm{CC}$. The mean inelasticity for energies of the order of $1 \mathrm{EeV}$ for neutrinonucleon interaction is $\langle y\rangle \approx 0.2$. It means that for NC interactions one hadronic EAS is produced on average with approximately $20 \%$ of the energy of the primary neutrino while for $\mathrm{CC}$ interactions there are three distinct cases for each neutrino flavor. We analyze the case when a tau-neutrino interacts and a tau is created which decay in a distance comparable to the size of the hadronic EAS generated by the neutrino interaction. Nearly $64 \%$ of the times the tau decay generates a hadronic EAS and $18 \%$ of the times an electromagnetic one. Approximately $17 \%$ of the times the tau decay in muon and neutrinos that do not generate any cascade.

We simulate tau-neutrino induced events for three differ- ent energies with $y$ values such that Double-Bang events have optimal characteristics to be observed. In the case of $E_{\mathrm{v}}=0.5 \mathrm{EeV}$ the mean inelasticity is compatible with the observation of the events because the average tau energy is $\left\langle E_{\tau}\right\rangle \simeq 0.4 \mathrm{EeV}$ what corresponds to a mean distance travelled before decaying of $\langle L\rangle \simeq 20 \mathrm{~km}$. For this specific values of $E_{\mathrm{v}}, y$, and $L$ the EAS that may be generated by the tau decay develops very close to the EAS created by the neutrinonucleon interaction, but at the same time both EASs are distinguishable. For the simulated energies of $E_{\mathrm{v}}=1.5 \mathrm{EeV}$ and $E_{\mathrm{v}}=5 \mathrm{EeV}$, if we consider just the mean inelasticity value, i.e., $y=0.2$, the energy of the tau is such that the tau decay does not happen inside the field of view of a supposed detector because on average it travels too long distance compared to the field of view before decaying. So we simulate events with values of $y$ above the average for the tau energy to correspond on average to a travel distance of $L \simeq 20 \mathrm{~km}$.

Therefore, we have made for this paper simulations for specific cases of primary neutrino energy and inelasticity, and used the mean values of tau decay length and the energy from the tau that goes to the second hadronic shower in a DoubleBang event. What happens if we consider the general case when the average values can be the not real or not measured ones? In Fig. 5 we plot the probability for the tau decay in a 

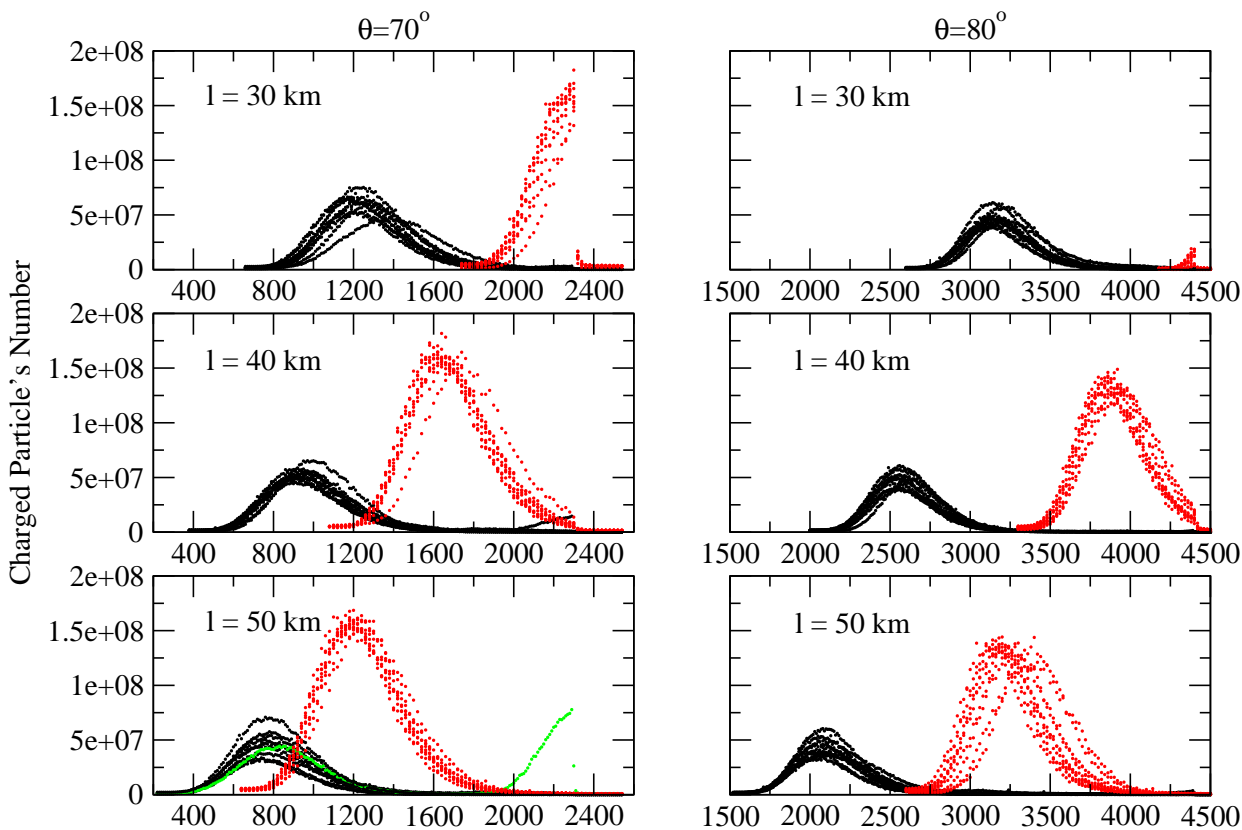

Depth $\left(\mathrm{g} / \mathrm{cm}^{2}\right)$

FIG. 3: Double-Bangs generated by tau-neutrinos in the atmosphere. Each graphic contains ten events represented by the number of charged particles as a function of depth with neutrino energy of $0.5 \mathrm{EeV}$. The incident angle is of $70^{\circ}$ in the first column and $80^{\circ}$ in the second one. The interaction distance to the ground is $l=30,40$ and $50 \mathrm{~km}$ respectively for each line. The first EASs, with less than $10^{8}$ charged particles, are represented in black and the second ones, due to the tau decay with more than the double number of particles, are represented in red. There is a fake Double-Bang event represented in green in the bottom left.

distance between 15 and $30 \mathrm{~km}$ after being created as a function of the inelasticity, for the different values of primary neutrino energy used in the simulations. Despite the mean inelasticity for these energies is approximately 0.2 , it has a very big dispersion. As can be seen in Fig. 7 of ref. [14], about $10 \%$ of the events are expected to be in the range of inelasticity $y=0.2 \pm 0.05,45 \%$ for $y<0.1$, and $30 \%$ for $y>0.3$. One sees from Fig. 5 that the simulations we present are for the cases of inelasticity when the probability of the tau decay in the range between 15 and $30 \mathrm{~km}$ is larger. Because approximately $70 \%$ of the events occur with inelasticity lower than 0.3 , among the cases analyzed in this paper, the most probable events are generated by neutrinos with energy of $E_{\mathrm{v}}=0.5 \mathrm{EeV}$.

It is important to notice that the $y$ distribution of the cross section is very dependent on the low- $x$ behavior of the parton distributions in a region beyond the access of accelerator experiments [41], so if the $x$ behavior is not the expected by the extrapolation of the parameters of the Standard Model, the $y$ dispersion may be different and our conclusions too. The Double-Bang events have the energy of the two EASs directly related to the inelasticity, as can be seen in Eqs. (4)-(5), and the detection os these kind of events can be of great importance to study the behavior of $y$ at ultra-high energies.

If the Double-Bang event characteristics like inelasticity and tau decay length follow the average, the energy range of the primary neutrino must be very strict because to observe the two EASs the primary neutrino energy cannot exceed about $1 \mathrm{EeV}$. But also the efficiency of the detector is an important factor and energies bellow $1 \mathrm{EeV}$ are not optimal for the FDs to observe events. Because the dispersion specially in the inelasticity and tau decay length, the primary neutrino energy range can be relaxed. The simulations we present give us a sign that the profile of Double-Bang events may be observed by FDs such as those of the Pierre Auger Observatory or Telescope Array. We also suggest that ground arrays such as the one of the Telescope Array and the Auger array may be another possibility to detect Double-Bangs. Almost horizontal Double-Bangs may develop both EASs inside the arrays. And finally the other possibility could be to use both techniques, for example, observing the first shower with the FD and the second, due to the tau decay, with the ground array.

We have studied the parameter space in which DoubleBang events may be measured by FDs. Taking the expected average values for the inelasticity and tau decay length at ultra-high energies, if the neutrino interaction happens between 30 and $50 \mathrm{~km}$ far from the detector, the incident angle must be bigger than $50^{\circ}$ and the most favorable to detect the events is around $75^{\circ}$ (see Figs. 2, 3, and 4). From Fig. 5 


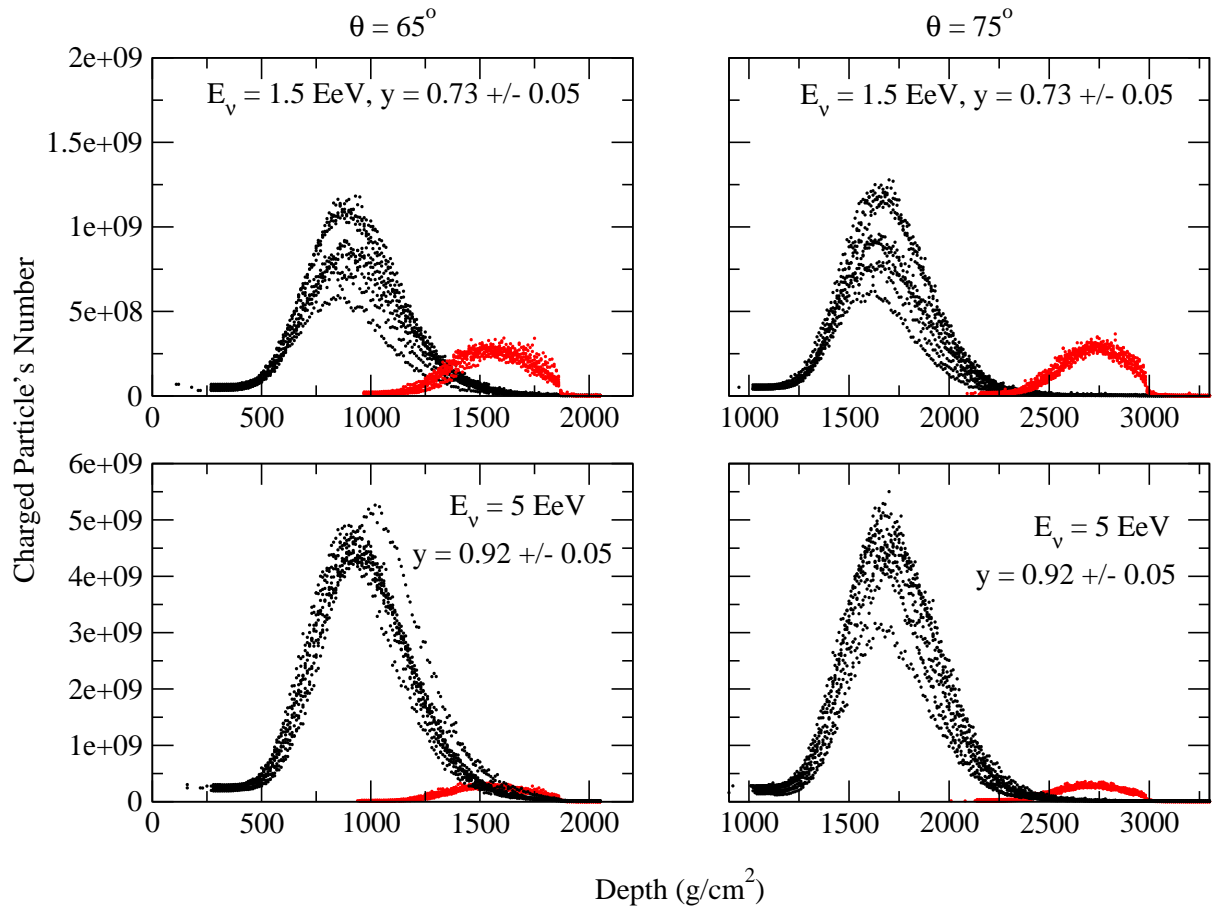

FIG. 4: Double-Bangs generated by tau-neutrinos in the atmosphere. Each graphic contains ten events represented by the number of charged particles as a function of depth generated $35 \mathrm{~km}$ far from the detector. The incident angle is of $65^{\circ}$ in the first column and $75^{\circ}$ in the second one. The neutrino energy is $1.5 \mathrm{EeV}$ for the graphics in the first line and $5 \mathrm{EeV}$ for the ones in the second line. The first EASs, with bigger number of charged particles, are represented in black while the second ones, due to the tau decay, are represented in red.

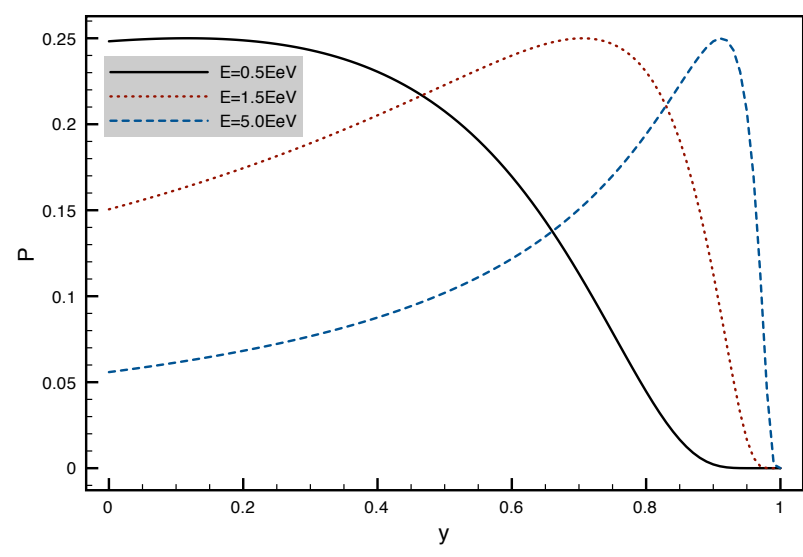

FIG. 5: Probability for the tau decay in a distance between 15 and $30 \mathrm{~km}$ after being created as a function of the inelasticity, for different values of primary neutrino energy. we learn that considering the inelasticity for the neutrino DIS cross section around 0.2 , the primary neutrino energy around $0.5 \mathrm{EeV}$ is optimal to detect Double-Bangs. In this sense, the HEAT extension of the Auger experiment [42] and the TALE extension of the Telescope Array experiment [43] will be of great importance also in the study of Double-Bangs. Both experiments will have bigger efficiency and field of view for events with energies bellow $1 \mathrm{EeV}$.

\section{Acknowledgments}

We thank specially O. Pisanti for her help with the simulations and discussions. The work was supported by $\mathrm{CNPq}$, FAPESP and AlBan.
[1] P. Sokolsky, Introduction to Ultrahigh Energy Cosmic Ray Physics (Westview Press, 2004).

[2] T. K. Gaisser, Cosmic rays and particle physics (Cambridge
University Press, UK, 1990).

[3] T. Yamamoto, K. Mase, M. Takeda, N. Sakaki, and M. Teshima (AGASA), Astropart. Phys. 20, 405 (2004), astro-ph/0312275. 
[4] T. Yamamoto (Pierre Auger), AIP Conf. Proc. 842, 1016 (2006), astro-ph/0601035.

[5] A. Letessier-Selvon (Pierre Auger), AIP Conf. Proc. 815, 73 (2006), astro-ph/0510627.

[6] R. Abbasi et al. (High Resolution Fly's Eye), Astropart. Phys. 25, 93 (2006), astro-ph/0601091.

[7] E. Zas, New J. Phys. 7, 130 (2005), astro-ph/0504610.

[8] X. Bertou, P. Billoir, O. Deligny, C. Lachaud, and A. LetessierSelvon, Astropart. Phys. 17, 183 (2002), astro-ph/0104452.

[9] J. G. Learned and S. Pakvasa, Astropart. Phys. 3, 267 (1995), hep-ph/9405296.

[10] H. Athar, Chin. J. Phys. 42, 1 (2004), hep-ph/0308188.

[11] G. Miele, S. Pastor, and O. Pisanti, Phys. Lett. B 634, 137 (2006), astro-ph/0508038.

[12] C. Aramo et al., Astropart. Phys. 23, 65 (2005), astro$\mathrm{ph} / 0407638$.

[13] S. Bottai and S. Giurgola, Astropart. Phys. 18, 539 (2003), astro-ph/0205325.

[14] D. Gora, M. Roth, and A. Tamburro, Astropart. Phys. 26, 402 (2007).

[15] J. Abraham et al. (Pierre Auger), Nucl. Instrum. Meth. A 523, 50 (2004).

[16] J. Abraham et al. (Pierre Auger), Science 318, 938 (2007), arXiv:0711.2256 [astro-ph].

[17] V. Verzi (Pierre Auger), Nucl. Phys. Proc. Suppl. 165, 37 (2007).

[18] C. C. H. Jui (HiRes and TA), AIP Conf. Proc. 867, 183 (2006).

[19] M. M. Guzzo and C. A. Moura, Astropart. Phys. 25, 277 (2006), hep-ph/0504270

[20] W. M. Yao et al. (Particle Data Group ), J. Phys. G 33, 267 (2006).

[21] K. S. Capelle, J. W. Cronin, G. Parente, and E. Zas, Astropart. Phys. 8, 321 (1998), astro-ph/9801313.

[22] L. Anchordoqui, T. Han, D. Hooper, and S. Sarkar, Astropart. Phys. 25, 14 (2006), hep-ph/0508312.

[23] T. K. Gaisser, F. Halzen, and T. Stanev, Phys. Rept. 258, 173
(1995), hep-ph/9410384.

[24] R. Gandhi, C. Quigg, M. H. Reno, and I. Sarcevic (1996), hep$\mathrm{ph} / 9609516$.

[25] A. Dar and A. De Rujula, Phys. Rept. 405, 203 (2004), astro$\mathrm{ph} / 0308248$.

[26] V. S. Berezinsky and A. Vilenkin, Phys. Rev. D 62, 083512 (2000), hep-ph/9908257.

[27] D. Allard et al., JCAP 0609, 005 (2006), astro-ph/0605327.

[28] R. Engel, D. Seckel, and T. Stanev, Phys. Rev. D 64, 093010 (2001), astro-ph/0101216.

[29] E. M. Henley and J. Jalilian-Marian, Phys. Rev. D 73, 094004 (2006), hep-ph/0512220.

[30] M. M. Guzzo and C. A. Moura (2003), hep-ph/0312119.

[31] W. M. Yao et al. (Particle Data Group), J. Phys. G 33, 33 (2006).

[32] S. Jadach, Z. Was, R. Decker, and J. H. Kuhn, Comput. Phys. Commun. 76, 361 (1993).

[33] M. Ambrosio et al. (2003), astro-ph/0302602.

[34] D. Heck, G. Schatz, T. Thouw, J. Knapp, and J. N. Capdevielle (1998), fZKA-6019.

[35] G. Corcella et al., JHEP 01, 010 (2001), hep-ph/0011363.

[36] S. Ostapchenko, Phys. Rev. D 74, 014026 (2006), hep$\mathrm{ph} / 0505259$.

[37] H. Fesefeldt (1985), pITHA-85-02.

[38] S. Argiro et al. (Pierre Auger) (2005), presented at 29th International Cosmic Ray Conference, Pune, India, 3-11 Aug.

[39] T. K. Gaisser (1977), rapporteur Lecture presented at 15th Int. Cosmic Ray Conf., Plovdiv, Bulgaria, Aug 13-26.

[40] J. Pumplin et al., JHEP 07, 012 (2002), hep-ph/0201195.

[41] J. A. Castro Pena, G. Parente, and E. Zas, Phys. Lett. B 500, 125 (2001), hep-ph/0011043.

[42] H. O. Klages (Pierre Auger) (2007), prepared for 30th International Cosmic Ray Conference (ICRC 2007), Merida, Yucatan, Mexico, 3-11 Jul 2007.

[43] URL http://www.telescopearray.org/papers/ TALE2005.pdf . 